\title{
VANISHING OF COHOMOLOGY OVER COHEN-MACAULAY RINGS
}

\author{
LARS WINTHER CHRISTENSEN AND HENRIK HOLM
}

\begin{abstract}
A 2003 counterexample to a conjecture of Auslander brought attention to a family of rings - colloquially called AC rings - that satisfy a natural condition on vanishing of cohomology. Several results attest to the remarkable homological properties of AC rings, but their definition is barely operational, and it remains unknown if they form a class that is closed under typical constructions in ring theory. In this paper, we study transfer of the AC property along local homomorphisms of Cohen-Macaulay rings. In particular, we show that the AC property is preserved by standard procedures in local algebra. Our results also yield new examples of Cohen-Macaulay AC rings.
\end{abstract}

\section{INTRODUCTION}

Vanishing of Ext groups and functors play a crucial role in the study of rings and their modules. For a fixed module $M$, vanishing of $\operatorname{Ext}^{n}(M, N)$ for all modules $N$ and integers $n \gg 0$ says that $M$ has finite projective dimension, in which case the functors $\operatorname{Ext}^{n}(M,-)$ vanish for all $n>$ proj.dim $(M)$. Finiteness of proj.dim $\operatorname{dim}_{R}(M)$ for all finitely generated $R$-modules is a lot to ask from a ring - in the noetherian case it means that $R$ is regular. Auslander conjectured that for every finitely generated module $M$, also those of infinite projective dimension, there would be a sort of an upper bound for non-vanishing of the groups $\operatorname{Ext}^{n}(M, N)$; see [1, ch. V]. The exact conjecture is that every Artin algebra $R$ satisfies the following:

(AC) For every finitely generated $R$-module $M$ there exists an integer $b_{M} \geqslant 0$ such that for every finitely generated $R$-module $N$ one has: $\operatorname{Ext}_{R}^{n}(M, N)=0$ for $n \gg 0$ implies $\operatorname{Ext}_{R}^{n}(M, N)=0$ for $n>b_{M}$.

An integer $b_{M}$ with this property is called an Auslander bound for $M$; the least such bound may be perceived as a "latent projective dimension" of $M$.

An example, discovered by Jorgensen and Şega 17, disproved Auslander's conjecture. However, several classes of rings do satisfy Auslander's condition (AC), and remarkable homological properties that flow from (AC) have been uncovered, for example, in work of Huneke and Jorgensen [14; see also [10.

The counterexample in [17] is a commutative Gorenstein local ring, which is a finite dimensional algebra, and even Koszul. Nevertheless, the condition (AC) is satisfied by all commutative local rings that are complete intersection or Golod, and that is striking: with regard to homological characteristics these two classes of rings usually belong on opposite sides of the spectrum.

Date: 23 February 2012.

2010 Mathematics Subject Classification. 13D07, 13H10.

Key words and phrases. AC ring, AB ring, Auslander's condition, Ext-index, c.i. local homomorphism. 
Among commutative noetherian local rings, those that satisfy (AC) are emerging as a family with intriguing homological properties, albeit one whose position relative to the traditional classes of rings is not easily described. In this paper, we give new examples - explicit and abstract - of Cohen-Macaulay local rings that satisfy (AC).

From this point on, all rings considered are commutative, noetherian, and local. In particular, $(R, \mathfrak{m})$ is such a ring, and $\widehat{R}$ denotes its $\mathfrak{m}$-adic completion.

Most Cohen-Macaulay rings of finite CM type are Golod and, therefore, known from [17, prop. 1.4] to satisfy even a uniform version of Auslander's condition:

(UAC) There exists an integer $b \geqslant 0$ such that for all finitely generated $R$-modules

$M$ and $N$ one has: $\operatorname{Ext}_{R}^{n}(M, N)=0$ for $n \gg 0$ implies $\operatorname{Ext}_{R}^{n}(M, N)=0$ for $n>b$.

In Section 1 we show by a direct argument that every Cohen-Macaulay ring of finite CM type satisfies (UAC).

An explicit collection of new examples of rings that satisfy (UAC) is constructed via results about preservation of the (AC) and (UAC) properties under standard operations in local algebra - such as completion and reduction modulo regular elements. These results were advertised in [10, rmk. 5.7]; they are proved in Section 2 , and the examples follow in Section 3 ,

The canonical maps $R \hookrightarrow \widehat{R}$ and $R \rightarrow R /(x)$, where $x$ is a regular element, are archetypes of local homomorphisms of finite flat dimension; they are even c.i. homomorphisms in the sense of Avramov 4. A classical chapter of local algebra studies transfer of ring theoretic properties along local homomorphisms. In Section 4 we add to it as we prove, essentially, that the (AC) and (UAC) properties descend along homomorphisms of finite flat dimension and ascend along c.i. homomorphisms.

\section{Maximal Cohen-Macaulay modules}

For a finitely generated $R$-module $M$, the $n$th syzygy in its minimal free resolution is denoted $\operatorname{syz}_{n}^{R}(M)$. For $i>n \geqslant 0$ there are isomorphisms:

$$
\operatorname{Ext}_{R}^{i-n}\left(\operatorname{syz}_{n}^{R}(M), N\right) \cong \operatorname{Ext}_{R}^{i}(M, N) .
$$

Let $R$ be Cohen-Macaulay of dimension $d$. A finitely generated $R$-module $M$ is called maximal Cohen-Macaulay, abbreviated MCM, if the equality $\operatorname{depth}_{R} M=d$ holds. Every $d$ th syzygy of a finitely generated $R$-module is either 0 or an MCM module, and every syzygy of an MCM module is an MCM module; see [18, prop. (1.16) and (1.3)]. These facts together with the theory of MCM approximations, which is due to Auslander and Buchweitz [2], are central to this section.

1.1 Remark. Let $R$ be Cohen-Macaulay of dimension $d$. Dimension shifting (1.0.1) shows that $R$ satisfies (AC) if and only if every MCM $R$-module has an Auslander bound. Every MCM module is a (finite) direct sum of indecomposable MCM modules, so $R$ satisfies (AC) if and only if every indecomposable MCM $R$ module has an Auslander bound. In particular, $R$ satisfies (UAC) if and only if the indecomposable MCM $R$-modules have a common Auslander bound.

A Cohen-Macaulay ring $R$ is said to be of finite $C M$ type if there are only finitely many indecomposable MCM $R$-modules, up to isomorphism.

1.2 Theorem. A Cohen-Macaulay local ring of finite CM type satisfies (UAC). 
Proof. Let $R$ be Cohen-Macaulay of finite CM type. By Remark 1.1, it suffices to prove that each of the finitely many indecomposable MCM $R$-modules has an Auslander bound. For an MCM $R$-module $X$, let $n(X)$ be the number of distinct, up to isomorphism, indecomposable MCM $R$-modules that occur as direct summands of any one of the syzygies $\operatorname{syz}_{i}^{R}(X)$, for $i \geqslant 0$.

Let $M$ be an indecomposable MCM $R$-module. If $n(M)$ is 1 , then $M$ is either free of rank 1 or a direct summand of every syzygy module $\operatorname{syz}_{i}^{R}(M)$ for $i \geqslant 0$, whence it has Auslander bound 0; cf. (1.0.1). Let $t \geqslant 1$ and assume that every MCM $R$-module $X$ with $n(X) \leqslant t$ has an Auslander bound. Assume $n(M)=t+1$. If $M$ occurs as a direct summand of one of its syzygies, say, $\operatorname{syz}_{l}^{R}(M)$ for some $l \geqslant 1$, then - by uniqueness of minimal free resolutions - $M$ occurs as a summand of infinitely many syzygies, namely $\operatorname{syz}_{l m}^{R}(M)$ for all $m \in \mathbb{N}$; whence $M$ has Auslander bound 0 . If $M$ does not occur as a direct summand of $\operatorname{syz}_{i}^{R}(M)$ for any $i \geqslant 1$, then one has $n\left(\operatorname{syz}_{1}^{R}(M)\right) \leqslant t$. By the induction hypothesis, $\operatorname{syz}_{1}^{R}(M)$ has an Auslander bound, whence $M$ has one, again by dimension shifting (1.0.1).

The embedding dimension of $R$, edim $R$, is the minimal number of generators of its maximal ideal, and the codimension is the difference $\operatorname{codim} R=\operatorname{edim} R-\operatorname{dim} R$. The multiplicity e $(R)$ of a Cohen-Macaulay ring is at least $\operatorname{codim} R+1$; if equality holds, then $R$ is said to have minimal multiplicity.

1.3 Remark. Eisenbud and Herzog [1] raise the question whether every complete Cohen-Macaulay ring of finite CM type and dimension at least 2 has minimal multiplicity. Cohen-Macaulay rings of minimal multiplicity are Golod, see Avramov [3, prop. 5.2.4], and satisfy (UAC) for a strong reason, see [17, prop. 1.4].

1.4 Observation. Let $R$ be Cohen-Macaulay and assume that it has a dualizing module. By 2 , thm. A], every finitely generated $R$-module $N$ has a maximal CohenMacaulay approximation: an exact sequence of finitely generated $R$-modules,

$$
0 \longrightarrow I \longrightarrow Y \longrightarrow N \longrightarrow 0
$$

where $Y$ is MCM, and $I$ is of finite injective dimension. If $X$ is an MCM module, then one has $\operatorname{Ext}_{R}^{n}(X, I)=0$ for $n \geqslant 1$; see Yoshino [18, cor. (1.13)] and 2, cor. 6.4]. Thus, for all $n \geqslant 1$ there are isomorphisms

$$
\operatorname{Ext}_{R}^{n}(X, Y) \cong \operatorname{Ext}_{R}^{n}(X, N) \text {. }
$$

From (1.0.1) and the displays above, it is not hard to see that it suffices to verify the conditions (AC) and (UAC) on MCM modules. That is, $R$ satisfies (AC) if and only if it satisfies:

For every MCM $R$-module $X$ there exists $b_{X} \geqslant 0$ such that for every MCM $R$ module $Y$ one has: $\operatorname{Ext}_{R}^{n}(X, Y)=0$ for $n \gg 0$ implies $\operatorname{Ext}_{R}^{n}(X, Y)=0$ for $n>b_{X}$.

And $R$ satisfies (UAC) if and only if it satisfies:

There exists an integer $b \geqslant 0$ such that for all MCM $R$-modules $X$ and $Y$ one has: $\operatorname{Ext}_{R}^{n}(X, Y)=0$ for $n \gg 0$ implies $\operatorname{Ext}_{R}^{n}(X, Y)=0$ for $n>b$.

\section{Completion and Reduction modulo Regular sequences}

Let $R$ be Cohen-Macaulay; we can now show that $R$ and $\widehat{R}$ satisfy (AC) simultaneously and - in a generalization of [14, prop. 3.3.(1)] — that $R$ and $R /(x)$ satisfy (AC) simultaneously, if $x$ is a regular element. 
2.1 Lemma. Let $x$ be a regular element in $R$. If $R$ satisfies (AC/UAC), then $R /(x)$ satisfies (AC/UAC).

Proof. Let $M$ and $N$ be finitely generated $R /(x)$-modules. The change of rings spectral sequence [8, XVI. $\left.\S 5 .(2)_{3}\right]$

$$
\mathrm{E}_{2}^{p, q}=\operatorname{Ext}_{R /(x)}^{q}\left(\operatorname{Tor}_{p}^{R}(R /(x), M), N\right) \Rightarrow \operatorname{Ext}_{R}^{p+q}(M, N)
$$

has zero differentials on the second and all subsequent pages. For each $n \geqslant 0$ it follows from [8, prop. 5.5] that there is an exact sequence

$$
0 \rightarrow \operatorname{Ext}_{R /(x)}^{n-1}(M, N) \rightarrow \operatorname{Ext}_{R}^{n}(M, N) \rightarrow \operatorname{Ext}_{R /(x)}^{n}(M, N) \rightarrow 0 .
$$

If $b$ is an Auslander bound for $M$ as an $R$-module, then it follows that $\max \{0, b-1\}$ is an Auslander bound for $M$ as an $R /(x)$-module.

A slightly different argument for the result above appeared in the proof of [14, prop. 3.2.(1)]; the converse for Cohen-Macaulay local rings is established in 2.4

The remaining proofs in this section use computations in the derived category over $R$; first we recall some notation. A complex of $R$-modules is graded cohomologically,

$$
M=\cdots \longrightarrow M^{n-1} \stackrel{\partial_{M}^{n-1}}{\longrightarrow} M^{n} \stackrel{\partial_{M}^{n}}{\longrightarrow} M^{n+1} \longrightarrow \cdots .
$$

The suspension of $M$ is the complex $\Sigma M$ with $(\Sigma M)^{n}=M^{n+1}$ and $\partial_{\Sigma M}=-\partial_{M}$. The $n$th cohomology module of $M$ is denoted $\mathrm{H}^{n} M$. Isomorphisms in the derived category over $R$ are marked by the symbol $\simeq$; they induce isomorphisms at the level of homology. We use the standard notation, $\mathbf{R H o m}_{R}(-,-)$ and $-\otimes_{R}^{\mathbf{L}}-$, for the right derived Hom functor and the left derived tensor product functor. For all $R$-modules $M$ and $N$ and all integers $n$ there are isomorphisms

$$
\operatorname{Ext}_{R}^{n}(M, N) \cong \mathrm{H}^{n} \mathbf{R} \operatorname{Hom}_{A}(M, N) \text { and } \operatorname{Tor}_{n}^{R}(M, N) \cong \mathrm{H}^{-n}\left(M \otimes_{R}^{\mathbf{L}} N\right) .
$$

In the next work-hose lemma, the ring $S$ need not be local.

2.2 Lemma. Let $(R, \mathfrak{m})$ be Cohen-Macaulay and assume that it has a dualizing module. Let $S$ be an $R$-algebra of finite flat dimension and with $S / \mathfrak{m} S \neq 0$. If $S$ satisfies (AC/UAC), then $R$ satisfies (AC/UAC).

Proof. By Observation 1.4 it suffices to consider cohomology of MCM $R$-modules. For such modules $X$ and $Y$ there are isomorphisms in the derived category over $R$,

$$
\begin{aligned}
\mathbf{R H o m}_{R}(X, Y) \otimes_{R}^{\mathbf{L}} S & \simeq \mathbf{R H o m}_{S}\left(X \otimes_{R}^{\mathbf{L}} S, Y \otimes_{R}^{\mathbf{L}} S\right) \\
& \simeq \mathbf{R H o m}_{S}\left(X \otimes_{R} S, Y \otimes_{R} S\right) .
\end{aligned}
$$

The first isomorphism is tensor evaluation, see Avramov and Foxby [6, lem. 4.4.(F)], and adjointness of Hom and tensor. The second isomorphism follows as one has $\operatorname{Tor}_{n}^{R}(X, S)=0=\operatorname{Tor}_{n}^{R}(Y, S)$ for $n \geqslant 1$; see [9, thm. (3.4)]. These isomorphisms and the assumption $S / \mathfrak{m} S \neq 0$ yield:

$$
\sup \left\{n \mid \operatorname{Ext}_{R}^{n}(X, Y) \neq 0\right\}=\sup \left\{n \mid \operatorname{Ext}_{S}^{n}\left(X \otimes_{R} S, Y \otimes_{R} S\right) \neq 0\right\} ;
$$

see Foxby [12, lem. 2.1.(2)]. Thus, if $b$ is an Auslander bound for the $S$-module $X \otimes_{R} S$, then $b$ is also an Auslander bound for the $R$-module $X$. 
2.3 Theorem. Let $(R, \mathfrak{m})$ be Cohen-Macaulay. Any one of the local rings

$$
R, \widehat{R}, R \llbracket X \rrbracket, \text { and } R[X]_{(\mathfrak{m}, X)}
$$

satisfies (AC/UAC) if and only if they all satisfy (AC/UAC).

Proof. The rings $R, \widehat{R}, R \llbracket X \rrbracket$, and $R[X]_{(\mathfrak{m}, X)}$ are all local and Cohen-Macaulay.

We first argue that $R$ and $\widehat{R}$ satisfy (AC/UAC) simultaneously. If $\widehat{R}$ satisfies (AC/UAC), then so does $R$ by [10, prop. 5.5], as $\widehat{R}$ is a faithfully flat $R$-algebra. For the converse, assume that $R$ satisfies (AC/UAC), and let $\boldsymbol{x}=x_{1}, \ldots, x_{d}$ be a maximal $R$-regular sequence; its image in $\widehat{R}$ is a maximal $\widehat{R}$-regular sequence. There is an isomorphism of rings $R /(\boldsymbol{x}) \cong \widehat{R} /(\boldsymbol{x})$, and this ring is (AC/UAC) by Lemma 2.1. As $\widehat{R}$ has a dualizing module, it satisfies (AC/UAC) by Lemma 2.2 .

If $R \llbracket X \rrbracket$ or $R[X]_{(\mathfrak{m}, X)}$ satisfies (AC/UAC), then so does $R$ by Lemma 2.1 For the converse, assume that $R$ and, therefore, $\widehat{R}$ satisfies (AC/UAC). The local ring $\widehat{R} \llbracket X \rrbracket$, which is Cohen-Macaulay and has a dualizing module, satisfies (AC/UAC) by Lemma 2.2 applied to the surjection $\widehat{R} \llbracket X \rrbracket \rightarrow \widehat{R}$. The isomorphisms

$$
R\left[\widehat{X]_{(\mathfrak{m}, X)}} \cong \widehat{R} \llbracket X \rrbracket \cong \widehat{R \llbracket X \rrbracket}\right.
$$

and the assertion established in the first part of the proof now show that $R \llbracket X \rrbracket$ and $R[X]_{(\mathfrak{m}, X)}$ satisfy $(\mathrm{AC} / \mathrm{UAC})$.

2.4 Corollary. Let $R$ be Cohen-Macaulay and $\boldsymbol{x}=x_{1}, \ldots, x_{n}$ be an $R$-regular sequence. The ring $R /(\boldsymbol{x})$ satisfies (AC/UAC) if and only if $R$ satisfies (AC/UAC).

Proof. The "if" part is immediate from Lemma 2.1. For the converse, assume that $R /(\boldsymbol{x})$ satisfies (AC/UAC). It is a Cohen-Macaulay ring, so by Theorem 2.3 the completion $\widehat{R /(\boldsymbol{x})} \cong \widehat{R} /(\boldsymbol{x})$ satisfies (AC/UAC). As the image of $\boldsymbol{x}$ in $\widehat{R}$ is a regular sequence, the ring $\widehat{R}$ satisfies (AC/UAC) by Lemma 2.2. and then another application of 2.3 shows that $R$ satisfies (AC/UAC).

A Cohen-Macaulay ring reduces modulo a regular sequence to an artinian ring, and by Corollary 2.4 the two rings satisfy (AC/UAC) simultaneously. In a clear analogy, the conditions ( $\mathrm{AC}$ ) and (UAC) can be verified on modules of finite length:

2.5 Proposition. Let $R$ be Cohen-Macaulay. It satisfies (AC) if and only if it satisfies the condition:

For every $R$-module $K$ of finite length there exists an integer $b_{K} \geqslant 0$ such that for every $R$-module $L$ of finite length one has: $\operatorname{Ext}_{R}^{n}(K, L)=0$ for $n \gg 0$ implies $\operatorname{Ext}_{R}^{n}(K, L)=0$ for $n>b_{K}$.

Furthermore, $R$ satisfies (UAC) if and only if it satisfies the condition:

There exists an integer $b \geqslant 0$ such that for all $R$-modules $K$ and $L$ of finite length one has: $\operatorname{Ext}_{R}^{n}(K, L)=0$ for $n \gg 0$ implies $\operatorname{Ext}_{R}^{n}(K, L)=0$ for $n>b$.

Proof. Assume that $R$ satisfies (AC/UAC) for modules of finite length. For $\widehat{R}$ modules $K$ and $L$ of finite length and integers $n \geqslant 0$ there are isomorphisms

$$
\operatorname{Ext}_{\widehat{R}}^{n}(K, L) \cong \operatorname{Ext}_{\widehat{R}}^{n}\left(\widehat{R} \otimes_{R} K, L\right) \cong \operatorname{Ext}_{R}^{n}(K, L) .
$$

As $R$-modules, $K$ and $L$ also have finite length; hence it follows that $\widehat{R}$ satisfies (AC/UAC) for modules of finite length. In view of Theorem 2.3 we can now assume that $R$ is complete and, in particular, that it has a dualizing module. 
By Observation 1.4 it is sufficient to prove that $R$ satisfies (AC/UAC) for MCM modules. Let $X$ and $Y$ be MCM $R$-modules, and let $\boldsymbol{x}=x_{1}, \ldots, x_{d}$ be a maximal $R$-regular sequence. As $\boldsymbol{x}$ is regular on $X$ and $Y$, there are isomorphisms

$$
X / \boldsymbol{x} X \simeq X \otimes_{R}^{\mathbf{L}} R /(\boldsymbol{x}) \text { and } Y / \boldsymbol{x} Y \simeq Y \otimes_{R}^{\mathbf{L}} R /(\boldsymbol{x})
$$

in the derived category over $R$. Nakayama's lemma, see [12, lem. 2.1.(2)], yields the first equality below, while the second follows from [6, lem. 4.4.(F)] and (11).

$$
\begin{aligned}
\sup \left\{n \mid \mathrm{H}^{n}\right. & \left.\mathbf{R H o m}_{R}(X, Y) \neq 0\right\} \\
& =\sup \left\{n \mid \mathrm{H}^{n}\left(\mathbf{R} \operatorname{Hom}_{R}(X, Y) \otimes_{R}^{\mathbf{L}} R /(\boldsymbol{x})\right) \neq 0\right\} \\
& =\sup \left\{n \mid \mathrm{H}^{n} \mathbf{R} \operatorname{Hom}_{R}(X, Y / \boldsymbol{x} Y) \neq 0\right\} .
\end{aligned}
$$

From (1) and adjointness of Hom and tensor one gets

$$
\mathbf{R H o m}_{R}(X / \boldsymbol{x} X, Y / \boldsymbol{x} Y) \simeq \mathbf{R H o m}_{R}\left(X, \mathbf{R H o m}_{R}(R /(\boldsymbol{x}), Y / \boldsymbol{x} Y)\right) .
$$

The Koszul complex on $\boldsymbol{x}$ is a free resolution of $R /(\boldsymbol{x})$, so there is an isomorphism

$$
\Sigma^{d} \mathbf{R H o m}_{R}(R /(\boldsymbol{x}),-) \simeq-\otimes_{R}^{\mathbf{L}} R /(\boldsymbol{x}) .
$$

The next equalities follow from (2) and Nakayama's lemma, from [6, lem. 4.4.(F)] and (4), and from (3), respectively.

$$
\begin{aligned}
\sup \left\{n \mid \mathrm{H}^{n}\right. & \left.\mathbf{R H o m}_{R}(X, Y) \neq 0\right\} \\
& =\sup \left\{n \mid \mathrm{H}^{n}\left(\mathbf{R} \operatorname{Hom}_{R}(X, Y / \boldsymbol{x} Y) \otimes_{R}^{\mathbf{L}} R /(\boldsymbol{x})\right) \neq 0\right\} \\
& =\sup \left\{n \mid \mathrm{H}^{n} \mathbf{R H o m}_{R}\left(X, \boldsymbol{\Sigma}^{d} \mathbf{R} \operatorname{Hom}_{R}(R /(\boldsymbol{x}), Y / \boldsymbol{x} Y)\right) \neq 0\right\} \\
& =\sup \left\{n \mid \mathrm{H}^{n} \mathbf{R} \operatorname{Hom}_{R}(X / \boldsymbol{x} X, Y / \boldsymbol{x} Y) \neq 0\right\}-d .
\end{aligned}
$$

That is, one has

$$
\sup \left\{n \mid \operatorname{Ext}_{R}^{n}(X, Y) \neq 0\right\}=\sup \left\{n \mid \operatorname{Ext}_{R}^{n}(X / \boldsymbol{x} X, Y / \boldsymbol{x} Y) \neq 0\right\}-d .
$$

As the $R$-modules $X / \boldsymbol{x} X$ and $Y / \boldsymbol{x} Y$ have finite length, a straightforward argument finishes the proof, cf. proof of Lemma 2.2 .

\section{An example}

One can construct new examples of Cohen-Macaulay local rings that satisfy (AC/UAC) from existing ones through the process of adjoining power series variables and killing regular elements. To get examples not covered in the literature, see the list [10, A.1], the new ring $R$ should not be Golod or complete intersection (c.i.); and if $R$ is Gorenstein, then its codimension, $\operatorname{codim} R$, should be at least 5 , and its multiplicity, $\mathrm{e}(R)$, should be at least $\operatorname{codim} R+3$. Furthermore, $R$ should not be of finite CM type, cf. Theorem 1.2 .

3.1 Proposition. Let $Q$ be a Cohen-Macaulay local ring and assume that it satisfies (AC/UAC). For integers $d, n \geqslant 1$ and $s_{i} \geqslant 2$, the local ring

$$
R=Q \llbracket X_{1}, \ldots, X_{d}, Y_{1}, \ldots, Y_{n} \rrbracket /\left(Y_{1}^{s_{1}}, \ldots, Y_{n}^{s_{n}}\right)
$$

is Cohen-Macaulay with $\operatorname{codim} R=\operatorname{codim} Q+n$, and it satisfies (AC/UAC). Moreover, the following hold:

(a) $R$ is not of finite $C M$ type.

(b) If $Q$ is not c.i., then $R$ is not c.i. and $R$ is not Golod.

(c) If $Q$ has infinite residue field and one has $s_{i} \geqslant 4$ for some $i \in\{1, \ldots, n\}$, then the inequality $\mathrm{e}(R) \geqslant \operatorname{codim} R+3$ holds. 
Proof. Clearly, $R$ is Cohen-Macaulay with $\operatorname{codim} R=\operatorname{codim} Q+n$, and it is immediate from Theorem 2.3 and Corollary 2.4 that $R$ satisfies (AC/UAC).

(a): Let $\mathfrak{q}$ be the maximal ideal in $Q$. The image of $Y_{1}$ in $R_{\left(\mathfrak{q}, Y_{1}, \ldots, Y_{n}\right)}$ is a zerodivisor, so $R$ is not an isolated singularity; in particular, $R$ is not of finite CM type; see Huneke and Leuschke [15, cor. 2].

(b): Assume that $Q$ is not c.i. By [4, (5.10)] the $\operatorname{ring} R$ is not c.i. As $Q$ is singular, one has $\operatorname{codim} R=\operatorname{codim} Q+n \geqslant 2$. The $R$-module $R /\left(Y_{1}\right)$ has constant Betti numbers equal to 1. It follows from [3, thm. 5.3.3.(5)] that $R$ is not Golod.

(c): Let $\mathfrak{a}$ be a minimal reduction of the maximal ideal $\mathfrak{q}$ in $Q$. It is elementary to verify that $\mathfrak{b}=\left(\mathfrak{a}, X_{1}, \ldots, X_{d}\right)$ is a reduction of $\mathfrak{m}=\left(\mathfrak{q}, X_{1}, \ldots, X_{d}, Y_{1}, \ldots, Y_{n}\right)$, the maximal ideal in $R$. Assume that $Q / \mathfrak{q}$ is infinite. It follows that $\mathfrak{a}$ is minimally generated by $\operatorname{dim} Q$ elements and, therefore, $\mathfrak{b}$ is generated by $\operatorname{dim} Q+d=\operatorname{dim} R$ elements. Hence, $\mathfrak{b}$ is a minimal reduction of $\mathfrak{m}$; see Swanson and Huneke [16, prop. 8.3.7 and cor. 8.3.5.(1)]. As $R$ is Cohen-Macaulay, [16, prop. 11.2.2] yields $\mathrm{e}(R)=$ length $R / \mathfrak{b}$. If one has $s_{i} \geqslant 4$ for some $i$, then $(\mathfrak{m} / \mathfrak{b})^{3}$ is non-zero, whence

$$
\text { length } R / \mathfrak{b} \geqslant \operatorname{edim} R / \mathfrak{b}+3 \geqslant \operatorname{edim} R-\operatorname{dim} R+3=\operatorname{codim} R+3 \text {. }
$$

We thank Louiza Fouli for clarifying for us the basics of multiplicities that are used in the proof of part (c) above.

All assumptions on $Q$ in Proposition 3.1 are satisfied by the ring in Example 3.2 thus for all choices of $d, n \geqslant 1$ and $s_{i} \geqslant 2$ the resulting ring $R$ in 3.1 is not among the rings on the list [10, A.1] nor is it of finite CM type.

3.2 Example. Let $k$ be a field of characteristic 0 and consider the local $k$-algebra

$$
Q=k[U, V, W] /\left(U^{2}-V^{2}, V^{2}-W^{2}, U V, V W\right) .
$$

It has radical cube zero and $\operatorname{edim} Q=3$, so it satisfies (UAC) by [17, prop. 1.1.(3)]. Moreover, $Q$ is not Gorenstein; in particular, it is not c.i.

Through a result of Heitmann [13, the construction in Proposition 3.1 also provides examples of unique factorization domains that satisfy (AC/UAC).

3.3 Observation. Let $Q$ be a complete Cohen-Macaulay local ring that satisfies (AC/UAC). Assume that $Q$ is not c.i. and that no integer is a zero divisor in $Q$; the ring in 3.2 serves as an example.

Let $R$ be as in Proposition 3.1. If $Q$ is not artinian or $d$ is at least 2, then $R$ has depth at least 2. By [13, thm. 8], there is a local unique factorization domain, $D$, with $\widehat{D} \cong R$. It follows from Theorem 2.3 that $D$ satisfies (AC/UAC). Of course, $R$ is also the completion of the ring $Q\left[X_{1}, \ldots, X_{d}, Y_{1}, \ldots, Y_{n}\right] /\left(Y_{1}^{s_{1}}, \ldots, Y_{n}^{s_{n}}\right)$ localized at the irrelevant maximal ideal, but that ring is not a domain.

\section{LOCAL HOMOMORPHISMS}

Let $(R, \mathfrak{m})$ and $(S, \mathfrak{n})$ be local rings, and let $\varphi: R \rightarrow S$ be a local homomorphism, that is, a ring homomorphism with $\varphi(\mathfrak{m}) \subseteq \mathfrak{n}$. The semicompletion of $\varphi$ is its composite with the embedding $S \hookrightarrow \widehat{S}$; it is written $\grave{\varphi}: R \rightarrow \widehat{S}$. The semicompletion of $\varphi$ admits a Cohen factorization; that is, there exist local homomorphisms

$$
R \stackrel{\dot{\varphi}}{\longrightarrow} R^{\prime} \stackrel{\varphi^{\prime}}{\longrightarrow} \widehat{S},
$$

such that $\grave{\varphi}=\varphi^{\prime} \dot{\varphi}$, where $\dot{\varphi}$ is flat with regular closed fiber $R / \mathfrak{m} R^{\prime}$, the ring $R^{\prime}$ is complete, and $\varphi^{\prime}$ is surjective; see Avramov, Foxby, and Herzog [7, thm. (1.1)]. If 
$\varphi$ is of finite flat dimension - that is, $S$ has finite flat dimension as an $R$-module via $\varphi$ - then the surjection $\varphi^{\prime}$ is of finite projective dimension; see [7, (3.3)].

A Gorenstein local ring satisfies (AC) if and only if it satisfies (UAC); following 14. such a ring is called an $A B$ ring. The (AC) and (UAC) properties descend along local homomorphisms of finite flat dimension:

4.1 Theorem. Let $\varphi: R \rightarrow S$ be a local homomorphism of finite flat dimension.

(a) If $S$ is Cohen-Macaulay and satisfies (AC/UAC), then $R$ is Cohen-Macaulay and satisfies (AC/UAC).

(b) If $S$ is an $A B$ ring, then $R$ is an $A B$ ring.

Proof. Assume that $S$ is Cohen-Macaulay and satisfies (AC/UAC), then $\widehat{S}$ has the same properties; see Theorem 2.3. Consider a Cohen factorization $R \rightarrow R^{\prime} \rightarrow \widehat{S}$ of the semicompletion $\dot{\varphi}$. By [7, (3.10)] the rings $R^{\prime}$ and $R$ are Cohen-Macaulay. The $\operatorname{ring} R^{\prime}$ is complete, hence it has a dualizing module, and by Lemma 2.2 it satisfies (AC/UAC). Finally, $R$ satisfies (AC/UAC) by [10, prop. 5.5]; this proves part (a). For part (b) it suffices to note that if $S$ is Gorenstein, then $R$ is Gorenstein; see Avramov and Foxby [5, thm. (2.4)].

The notation $(R, \mathfrak{m}, k)$ specifies that $k$ is the residue field of the local ring $(R, \mathfrak{m})$.

4.2 Lemma. Let $\varphi:(R, \mathfrak{m}, k) \rightarrow(S, \mathfrak{n}, k)$ be a local homomorphism of complete $k$-algebras. If $R$ is Cohen-Macaulay and satisfies (AC/UAC), then $\varphi$ has a Cohen factorization $R \rightarrow R^{\prime} \rightarrow S$, where $R^{\prime}$ is Cohen-Macaulay and satisfies (AC/UAC).

Proof. Let $y_{1}, \ldots, y_{n}$ be a generating set for $\mathfrak{n}$. As the field $k$ is a Cohen ring for $S$, in the sense of [7, (1.0.2)], the inclusion $k \hookrightarrow S$ extends by the assignment $Y_{i} \mapsto y_{i}$ to a surjective homomorphism $\pi: k \llbracket Y_{1}, \ldots, Y_{n} \rrbracket \rightarrow S$. Set $R^{\prime}=R \llbracket Y_{1}, \ldots, Y_{n} \rrbracket$, then $R^{\prime}$ is complete and a flat local extension of $R$ with regular closed fiber $R^{\prime} / \mathfrak{m} R^{\prime} \cong$ $k \llbracket Y_{1}, \ldots, Y_{n} \rrbracket$. The homomorphism $\varphi$ extends by the assignment $Y_{i} \mapsto y_{i}$ to a homomorphism $R^{\prime} \rightarrow S$; it is surjective because $\pi$ is surjective. Thus, $R \hookrightarrow R^{\prime} \rightarrow S$ is a Cohen factorization of $\varphi$. If $R$ is Cohen-Macaulay and satisfies (AC/UAC), then $R^{\prime}$ has the same properties; see Theorem 2.3 .

Following [4, 3.1], a local homomorphism $\varphi: R \rightarrow S$ is called c.i., for complete intersection, if there is a Cohen factorization $R \rightarrow R^{\prime} \rightarrow \widehat{S}$ of the semicompletion $\grave{\varphi}$ in which the kernel of the surjection $R^{\prime} \rightarrow S$ is generated by an $R^{\prime}$-regular sequence.

Here is an ascent result for the (AC) and (UAC) properties:

4.3 Theorem. Let $\varphi:(R, \mathfrak{m}, k) \rightarrow(S, \mathfrak{n}, k)$ be a c.i. local homomorphism of $k$ algebras.

(a) If $R$ is Cohen-Macaulay and satisfies (AC/UAC), then $S$ is Cohen-Macaulay and satisfies (AC/UAC).

(b) If $R$ is an $A B$ ring, then $S$ is an $A B$ ring.

Proof. Assume that $R$ is Cohen-Macaulay and satisfies (AC/UAC), then $\widehat{R}$ has the same properties; see Theorem 2.3. By Lemma 4.2 the semicompletion $\grave{\varphi}$ has a Cohen factorization $R \rightarrow R^{\prime} \rightarrow \widehat{S}$, in which $R^{\prime}$ is Cohen-Macaulay and satisfies (AC/UAC). As $\varphi$ is c.i., the kernel of the surjection $R^{\prime} \rightarrow \widehat{S}$ is generated by an 
$R^{\prime}$-regular sequence; see [4, (3.3)]. It follows from Corollary 2.4 that $\widehat{S}$ is CohenMacaulay and satisfies (AC/UAC) and, therefore, $S$ is Cohen-Macaulay and satisfies (AC/UAC); see Theorem 2.3. This proves part (a).

For part (b), recall that c.i. homomorphisms are Gorenstein by [5, thm. (2.4)]. Thus, if $R$ is Gorenstein, it then follows from [7, (3.11)] that $S$ is Gorenstein.

\section{REFERENCES}

[1] Maurice Auslander, Selected works of Maurice Auslander. Part 1, American Mathematical Society, Providence, RI, 1999, Edited and with a foreword by Idun Reiten, Sverre O. Smalø, and Øyvind Solberg. MR1674397

[2] Maurice Auslander and Ragnar-Olaf Buchweitz, The homological theory of maximal CohenMacaulay approximations, Mém. Soc. Math. France (N.S.) (1989), no. 38, 5-37, Colloque en l'honneur de Pierre Samuel (Orsay, 1987). MR1044344

[3] Luchezar L. Avramov, Infinite free resolutions, Six lectures on commutative algebra (Bellaterra, 1996), Progr. Math., vol. 166, Birkhäuser, Basel, 1998, pp. 1-118. MR1648664

[4] Locally complete intersection homomorphisms and a conjecture of Quillen on the vanishing of cotangent homology, Ann. of Math. (2) 150 (1999), no. 2, 455-487. MR1726700

[5] Luchezar L. Avramov and Hans-Bjørn Foxby, Gorenstein local homomorphisms, Bull. Amer. Math. Soc. (N.S.) 23 (1990), no. 1, 145-150. MR1020605

[6] - Homological dimensions of unbounded complexes, J. Pure Appl. Algebra 71 (1991), no. 2-3, 129-155. MR1117631

[7] Luchezar L. Avramov, Hans-Bjørn Foxby, and Bernd Herzog, Structure of local homomorphisms, J. Algebra 164 (1994), no. 1, 124-145. MR1268330

[8] Henri Cartan and Samuel Eilenberg, Homological algebra, Princeton Landmarks in Mathematics, Princeton University Press, Princeton, NJ, 1999, With an appendix by David A. Buchsbaum, Reprint of the 1956 original. MR1731415

[9] Lars Winther Christensen, Hans-Bjørn Foxby, and Anders Frankild, Restricted homological dimensions and Cohen-Macaulayness, J. Algebra 251 (2002), no. 1, 479-502. MR1900297

[10] Lars Winther Christensen and Henrik Holm, Algebras that satisfy Auslander's condition on vanishing of cohomology, Math. Z. 265 (2010), no. 1, 21-40. MR2606948

[11] David Eisenbud and Jürgen Herzog, The classification of homogeneous Cohen-Macaulay rings of finite representation type, Math. Ann. 280 (1988), no. 2, 347-352. MR929541

[12] Hans-Bjørn Foxby, Isomorphisms between complexes with applications to the homological theory of modules, Math. Scand. 40 (1977), no. 1, 5-19. MR0447269

[13] Raymond C. Heitmann, Characterization of completions of unique factorization domains, Trans. Amer. Math. Soc. 337 (1993), no. 1, 379-387. MR1102888

[14] Craig Huneke and David A. Jorgensen, Symmetry in the vanishing of Ext over Gorenstein rings, Math. Scand. 93 (2003), no. 2, 161-184. MR2009580

[15] Craig Huneke and Graham J. Leuschke, Two theorems about maximal Cohen-Macaulay modules, Math. Ann. 324 (2002), no. 2, 391-404. MR1933863

[16] Craig Huneke and Irena Swanson, Integral closure of ideals, rings, and modules, London Mathematical Society Lecture Note Series, vol. 336, Cambridge University Press, Cambridge, 2006. MR2266432

[17] David A. Jorgensen and Liana M. Şega, Nonvanishing cohomology and classes of Gorenstein rings, Adv. Math. 188 (2004), no. 2, 470-490. MR2087235

[18] Yuji Yoshino, Cohen-Macaulay modules over Cohen-Macaulay rings, London Mathematical Society Lecture Note Series, vol. 146, Cambridge University Press, Cambridge, 1990. MR1079937

Department of Math. and Stat., Texas Tech University, Lubbock, TX 79409, U.S.A.

E-mail address: lars.w.christensen@ttu.edu

$U R L:$ http://www.math.ttu.edu/ lchriste

Department of Basic Sciences and Environment, University of Copenhagen, Thorvaldsensvej 40, DK-1871 Frederiksberg C, Denmark

E-mail address: hholm@life.ku.dk

URL: http://www.matdat.life.ku.dk/ hholm 